\title{
Impact of nanoparticle size and surface chemistry on peptoid self-assembly
}

\author{
Madison Monahan ${ }^{\mathrm{a}}$, Micaela Homer ${ }^{\mathrm{a}}$, Shuai Zhang, ${ }^{\mathrm{b}, \mathrm{c}}$, Renyu Zheng ${ }^{\mathrm{c}, \mathrm{d}}$, Chun-Long Chen ${ }^{\mathrm{c}, \mathrm{d}}$, \\ James De Yoreo $^{\mathrm{a}, \mathrm{b}, \mathrm{c}}$, Brandi M. Cossairt ${ }^{\mathrm{a} *}$ \\ aDepartment of Chemistry, University of Washington, Box 351700, Seattle, WA 98195-1700. \\ ${ }^{b}$ Department of Materials Science and Engineering, University of Washington, Seattle, WA \\ 98195-1700. \\ 'Physical Sciences Division, Pacific Northwest National Laboratory, Richland, WA 99354. \\ dDepartment of Chemical Engineering, University of Washington, Seattle, WA 98195. \\ *cossairt@uw.edu
}

\begin{abstract}
.
Self-assembled organic nanomaterials can be generated by bottom-up assembly pathways where the structure is controlled by the organic sequence and altered using $\mathrm{pH}$, temperature, and solvation. In contrast, self-assembled structures based on inorganic nanoparticles typically rely on physical packing and drying effects to achieve uniform superlattices. By combining these two chemistries to access inorganic-organic nanostructures, we aim to understand the key factors that govern the assembly pathway and structural outcomes in hybrid systems. In this work, we outline two assembly regimes between quantum dots (QDs) and reversibly binding peptoids. These regimes can be accessed by changing the solubility and size of the hybrid (peptoid-QD) monomer unit. The hybrid monomers are prepared via ligand exchange, assembled, and resulting assemblies are studied using ex-situ transmission electron microscopy as a function of assembly time. In aqueous conditions, QDs were found to stabilize certain morphologies of peptoid intermediates and generate a unique final product consisting of multilayers of small peptoid sheets linked by QDs. The QDs were also seen to facilitate or inhibit assembly in organic solvents based on the relative hydrophobicity of the surface ligands, which ultimately dictated the solubility of the hybrid monomer unit. Increasing the size of the QDs led to large hybrid sheets with regions of highly ordered square-packed QDs. A second, smaller QD species can also be integrated to create binary hybrid lattices. These results create a set of design principles for controlling the structure and structural evolution of hybrid peptoid-QD assemblies and contribute to the predictive synthesis of complex hybrid matter.
\end{abstract}

\section{TOC Graphic.}

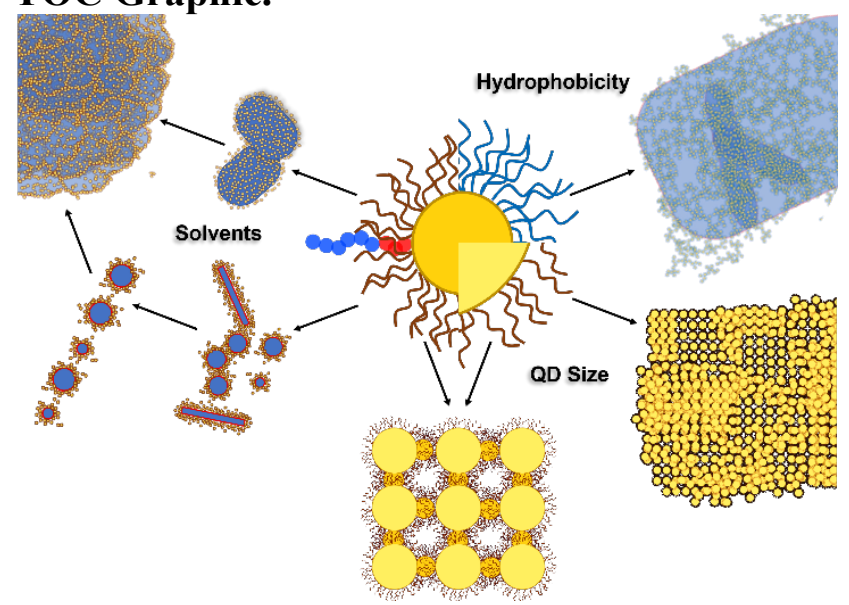




\section{Introduction.}

Hierarchically organized nanomaterials are often generated by mimicking natural systems wherein functional properties emerge due to order and organization across multiple length scales $^{1-3}$. To predictively generate such materials, we must understand and systematize the atomic-level interactions that lead to organization of the functional building blocks across length scales. Traditionally, hybrid organic-inorganic nanomaterials are synthesized via bottom-up assembly or through templated assembly on a scaffold ${ }^{4-7}$. In these approaches, organic molecules, which are typically DNA, peptides (or peptoids), polymers, or small multitopic organic molecules, are used as the structure-directing elements with covalent and non-covalent interactions dictating the possible assembly outcomes ${ }^{1,5-13}$. These interactions are dependent on the sequence used and can often be controlled using $\mathrm{pH}$, temperature, electrolytes, solvent, and/or pendant groups ${ }^{14-18}$. When inorganic nanoparticles act as the driving force for assembly, the generated structures are often a result of particle packing and drying effects. In these systems, the structures can be tuned through size, surface chemistry, ligand packing or morphology of the nanoparticles but frequently lack precision and are often limited to 2D films or layered structures of the aforementioned films ${ }^{19-23}$. Exquisite 3D hybrid structures have been achieved bottom-up using hydrogen bonding interactions of complimentary DNA strands to precisely control nanoparticle locations relative to one of another size or composition ${ }^{11-13}$. However, these systems are limited to assembly in aqueous conditions due to the stability of DNA, thus limiting the types of nanoparticles that can be used without significant surface modification. Coupling nanoparticles to information-rich linkers that are stable in organic media would offer the possibility of accessing new hybrid materials with unique functionality. Assemblies in organic media have been explored, typically with self-assembling polymers as the structure-directing agent and show promise to not only organize nanoparticles but to do in a predictive manner to access a wide variety of morphologies ${ }^{24-27}$.

Peptoids, or poly-N-substituted glycines, are peptide analogs that are functionalized on the nitrogen rather than carbon in the backbone sequence ${ }^{28-30}$. Through functionalization of the nitrogen, the monomers can be made soluble in a wide range of solvents, including organics, and the hydrogen-bonding interactions that often dominate secondary structure in peptides are absent ${ }^{28-30}$. Without hydrogen bonding, the structure can be tuned by the sequence of the peptoid monomer through side chain/side chain and side chain/backbone interactions. Peptoids have been shown to self-assemble into a variety of structures, most notably tubes, sheets, and helices ${ }^{31-35}$. Di-block peptoids containing a hydrophobic region and hydrophilic region are common selfassembling sequences that assemble in a mixed solvent system (1:1, water: organic solvent). In these systems, the slow evaporation of the organic solvent (initially used to solubilize the peptoid) acts as the driving force for assembly ${ }^{31,32}$. As the organic solvent evaporates, the environment becomes less favorable for the hydrophobic domain, resulting in a stacking effect where the peptoids interdigitate to form a bilayer structure with hydrophilic residues on the surface ${ }^{33,36,37}$.

The resulting peptoid structures have been explored as a function of sequence length, hydrophobicity, end group functionality, $\mathrm{pH}$, temperature, and as templates for nucleation and assembly of nanoparticles ${ }^{9,31,32,38-44}$ In previous work, we utilized peptoids as a scaffold for assembly of hybrid nanomaterials through post-synthetic addition of complimentary nanoparticles to pre-formed sheets and tubes to decorate the surface via a covalent linkage ${ }^{41}$. Specifically, we assembled CdSe nanoparticles onto peptoid tubes and sheets using a non- 
reversible thiol-maleimide click reaction that generates a covalent linkage between the nanoparticles and the assembled peptoid macrostructure. This approach allowed for control over the nanoparticle density as a function of the peptoid side-chain composition. Inorganic species have also been directly appended to the hydrophilic end of the peptoid monomers during the initial solid-phase synthesis, creating a hybrid peptoid monomer that can, in principle, then be assembled into larger-scale structure ${ }^{39}$. In this work, we demonstrate the versatility of a related approach by using peptoid monomers as ligands on quantum dots (QDs) to tune assembly pathways bottom up, with the final hybrid structures being a function of QD concentration, surface chemistry, and size.

CdSe and CdS QDs were attached to peptoid monomers to study the impact of these different pendant groups on assembly. The peptoid monomers contained six hydrophobic residues (N-[2-(4-bromophenyl)ethyl]glycine, Nbrpe $\left.{ }_{6} \mathrm{Dig}\right)$ and were terminated with a carboxylic acid (Scheme 1). This relatively short chain peptoid was chosen for its structural simplicity, diverse solubility properties, and limited steric bulk. A carboxylic acid group was chosen as the binding moiety due to the reversibility of the interaction with the QD surface, allowing for attachment through a facile carboxylic acid - carboxylate ligand exchange reaction ${ }^{45}$.

Furthermore, the dynamic interaction between the QD and the peptoid monomer provides a mechanism for the system to reach equilibrium and reduce the heterogeneity in the number of peptoids bound to a given QD. By starting with QDs capped with carboxylate ligands, we estimate an approximate equilibrium constant for the ligand exchange with peptoid of one ${ }^{45}$. For the QD composition, CdSe and CdS were chosen due to the depth of existing literature for these materials in diverse solvents, as well as the well-developed knowledge on ligand exchange and binding affinities for many different systems ${ }^{45-48}$. Cadmium chalcogenides are known to be relatively stable in water without significant surface modification ${ }^{46}$. Using QDs as pendant groups on the peptoids allows for straightforward modification of the peptoids without drastically modifying the synthetic chemistry or reactivity. This allows for a systematic study into how the pendant groups' size, hydrophobicity, and concentration alter the assembly process. This method further allows for a single peptoid sequence to provide access to a variety of structures without having to design a new sequence and introduces driving forces for assembly not accessible with peptoids alone.
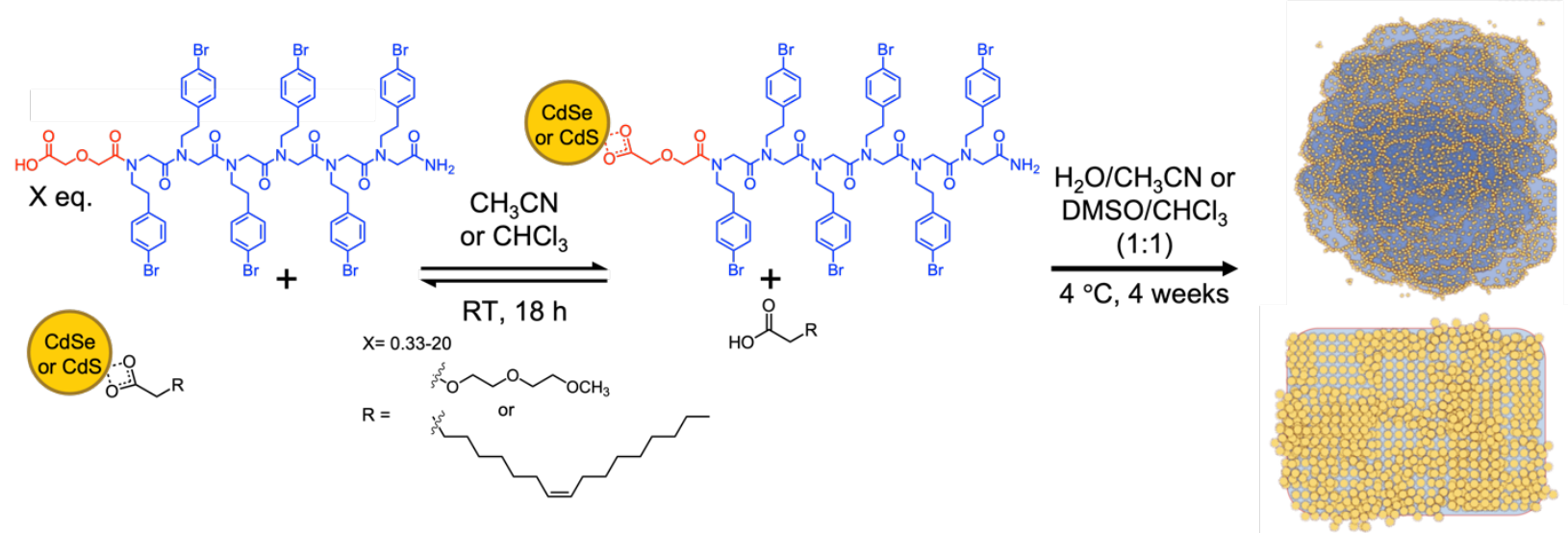

Scheme 1. General conditions for carboxylic acid (red)-terminated peptoid (blue) ligand exchange onto QDs (yellow) and assembly conditions for hybrid sheet formation. 


\section{Results and Discussion.}

Peptoid and hybrid material assembly as a function of the peptoid monomer:QD ratio.

To study assembly in a range of solvents, CdSe QDs $(2.4 \mathrm{~nm})$ were synthesized with 2[2-(2-methoxyethoxy)ethoxy)acetic acid (MEEAA) to generate QDs with a wide solubility range in both aqueous and organic solutions. Peptoid monomers were added to the QDs in varying concentrations (0.3-20 eq. of peptoid monomers per QD) and incubated for $18 \mathrm{~h}$ in $\mathrm{MeCN}$. Incubation for $18 \mathrm{~h}$ allowed the system to reach equilibrium and resulted in a partial ligand exchange, as evidenced by ${ }^{1} \mathrm{H}$ NMR (Figure S1). After $18 \mathrm{~h}$, water was added to the solution to initiate the assembly process. This solution was allowed to assemble at $4{ }^{\circ} \mathrm{C}$ for up to 4 weeks via slow evaporation of MeCN. Ex situ transmission electron microscopy (TEM) was the primary characterization mode, with scanning TEM (STEM) and bright field (BF) to identify the microstructures and nanostructures, respectively. The assembly process was tracked by taking aliquots from the reaction solution and preparing TEM grids. Initial samples were taken prior to the addition of water $(0 \mathrm{~h})$ to determine if any assembly occurred without anti-solvent addition. Image timepoints for the assemblies were taken relative to anti-solvent addition at $1 \mathrm{~h}, 24 \mathrm{~h}, 4-5$ $\mathrm{d}, 2 \mathrm{w}$, and $4 \mathrm{w}$.

Peptoid assembly was first tracked in the absence of QDs to gain a baseline for comparing intermediates arising during the assembly process. The peptoid partially dissolved in $\mathrm{MeCN}$ to produce non-uniform, elongated sheets and hollow spheres (Figure S2) that evolved into large, aggregated sheets after $18 \mathrm{~h}$ (Figure 1a). Water addition solubilized the peptoid, facilitating rearrangement into small sheets and micelles (Figure 1b,c). The spherical structures were assigned as micelles due to their solid nature and small size $(20.9 \pm 4.1 \mathrm{~nm})$. Over the course of 4 weeks, the micelles remained present while sheets continued to grow until rectangular sheets with straight edges were observed (Figure 1e).

Adding MEEAA-CdSe QDs into the peptoid solution prior to assembly produced a different final product and altered the nature of the intermediates. In MeCN, peptoid fibrils with QDs on the edges were observed rather than large peptoid-based aggregates (Figure 1f,k). With the addition of water, the nanofibers remained in addition to peptoid micelles with QDs on the edges (Figure 1g,l). Upon initial imaging, the peptoid-QD spheres appeared solid in STEM mode, but prolonged imaging led to hollow structures with high contrast exteriors (Figure S3a). The production of hollow structures implies a carbon-rich center that was destroyed by the electron beam. BF mode confirmed QDs only present on the edges, thus the assignment of micelle-like peptoid structure with QDs adhering to the surface (Figure S3b,c). The CdSe QDs were seen to etch upon exposure to the peptoids, resulting in a diameter of $1.9 \pm 0.2 \mathrm{~nm}$ that remained constant for up to 4 weeks in solution (Figure S4). After $24 \mathrm{~h}$, the QD-decorated micelles were seen to arrange into a linear pattern with an average spacing of $89.4 \pm 51.6 \mathrm{~nm}$ (Figure 1h). The distance between hybrid micelles was too large to correspond to one or two peptoid monomers, therefore we hypothesize a thin peptoid ribbon or fiber connecting the structures that was not easily seen next to the high contrast QDs (Figure 1m). Over the course of 4 weeks, the connected micelles began to ripen through bending of the linear structures (Figure S5) to form small, QD-covered sheets (Figure 1i,n), reminiscent of structures formed upon addition of QDs to pre-formed sheets ${ }^{41}$. These small hybrid peptoid/QD sheets eventually aggregated to form large multilayer aggregates $(>5 \mu \mathrm{m}$, Figure $\mathbf{1 j}, \mathbf{0})$. This final hybrid multilayer material contained small QD-decorated peptoid sheets similar to those observed earlier in the assembly, but with a higher density of QDs on the edges (Figure S6). We propose that these QDs act as linkers to bind the small sheets together into one large assembly, as has 
been previously observed for silica-peptoid hybrid structures ${ }^{42}$. This series of experiments demonstrate the synthesis of hybrid materials in which both the QDs and the peptoid operate synergistically to dictate the final structure.
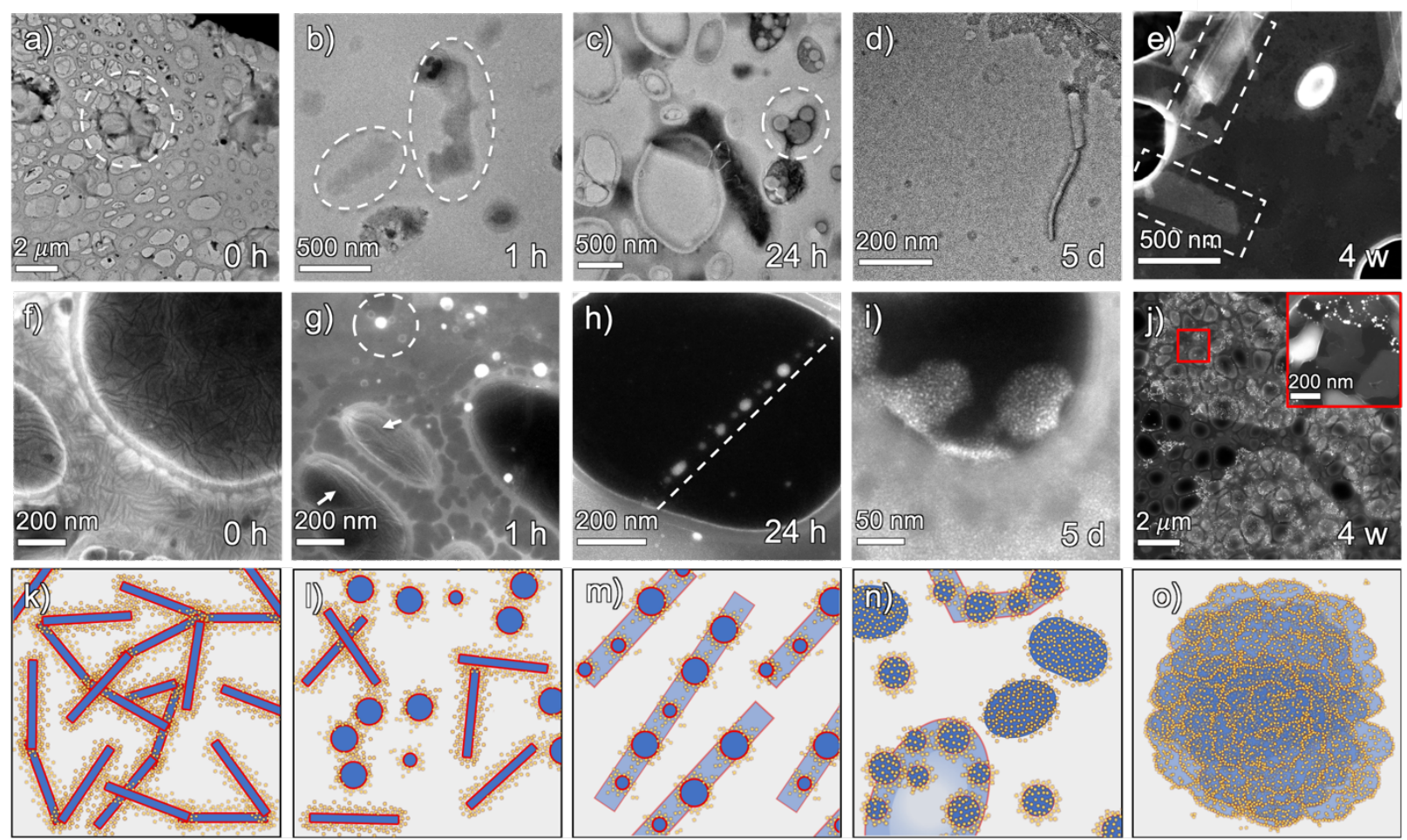

Figure 1. (a-e) Peptoid assembly without QDs in $\mathrm{H}_{2} \mathrm{O} / \mathrm{MeCN}$. Without water, large sheet-like aggregates are seen (a) that dissolved and began to form small sheets upon water addition (b). Peptoid micelles (c) are seen throughout the assembly process as the sheets grow to be welldefined (d). After 4 weeks, large crystalline sheets are seen (e). Peptoid edges are denoted by white dashed lines. (f-o) Assembly over time of MEEAA-CdSe QDs with 1 eq. of peptoid in $\mathrm{H}_{2} \mathrm{O} / \mathrm{MeCN}$. Peptoid fibrils with QDs on the edges are seen before and right after water addition (f,g white arrows). Peptoid micelles with QDs on the edges are also observed ( $g$ ) that tend towards linear arrangements $(\mathrm{h})$. After 5 days, small peptoid sheets are seen with a uniform QD distribution (i). Finally, the QDs are seen to concentrate at the sheet edges and facilitate the formation of large multilayer aggregates (j) composed of many small sheets (red inset, j). Peptoid edges are denoted with white dashed lines. Schematic representation of the assembly process (ko) with QDs in yellow, peptoid backbone in blue, and peptoid carboxylic acid in red.

The importance of the peptoid in this system was investigated by changing the number of peptoid monomers relative to the CdSe QD and changing the sequence of peptoid used. 20-0.33 equivalents of peptoid relative to QD were investigated while keeping the peptoid concentration constant to allow for direct comparison of assemblies at a given time point. At high peptoid equivalents, the system was seen to phase segregate with minimal integration of QDs into the structure. Here we propose the QDs are acting as defects, causing rolling and elongation of the sheets after assembly (Figure S7a). Low peptoid equivalents (5-0.33 eq.) led to assembly pathways with the same intermediates and end products as the one equivalent case described above (Figure S7b-e). However, the speed of the assembly and the stage at which different intermediates appeared varied in a consistent manner based on peptoid equivalents (Figure S8). 
In general, we observed a decrease in the overall rate of progression at higher peptoid equivalents. We hypothesize this decrease in assembly speed is due to a decrease in diffusion of the hybrid peptoid-QD monomers as the number of peptoids on the surface of the QDs and the size of the hybrid unit increases.

Changing the sequence of the peptoid to an asymmetric sequence with alternating (4bromophenyl)ethyl and (4-bromophenyl)methyl side chain groups, (NbrpeNbrpm) 3 Dig (Figure S9a), which forms twisted nanoribbons in absence of QDs, yielded inconsistent structures both within a given TEM grid and across samples regardless of timepoint or peptoid equivalents (Figure S9). However, alteration of the assembly pathway with QDs was seen and gave structures distinct from the peptoid alone. The assemblies showed very large hybrid materials as early as $1 \mathrm{~h}$ for all samples but did not show any distinguishable trends. It is hypothesized that the asymmetric sequence forms a twisted structure due to the differences in packing between the two sides of the peptoid ${ }^{31,32,36,37}$. By having a bulky QD at the end of the sequence, this difference in packing could be disrupted and the extent of the disruption may depend on how many peptoids are on a given QD, yielding inconsistent products. As a result, the rest of the studies described below were performed with the symmetric peptoid (Nbrpe ${ }_{6} \mathrm{Dig}$ ) at 1 eq. per QD.

Modulating $Q D$ surface chemistry to improve assembly in organic solvents.

By changing the ligand on the QD surface we can directly probe how the hydrophobicity of the peptoid-QD unit impacts the assembly process, allowing us to determine how different design elements lead to changes in the reaction landscape and ultimately the structure of the final assemblies. CdSe QDs $(2.4 \mathrm{~nm})$ were synthesized with oleic acid (OA) and MEEAA to generate hydrophobic and amphiphilic QDs, respectively, while maintaining carboxylate binding groups. To better solubilize the OA-CdSe QDs, the mixtures were assembled in dimethyl sulfoxide (DMSO) and chloroform $\left(\mathrm{CHCl}_{3}\right)$. Chloroform was chosen to dissolve the OA-CdSe QDs and peptoid monomers, replacing acetonitrile as the evaporating solvent, and DMSO to replace water as the higher boiling point polar solvent to create unfavorable interactions with the aryl halogens on the peptoid. The peptoid monomer was assembled alone in these solvents to get a baseline for peptoid assembly. In $\mathrm{CHCl}_{3}$, the peptoids formed a mixture of large fibers and small rectangular sheets immediately after dissolution (Figure S10). After $18 \mathrm{~h}$, only aggregates of large hollow spheres several hundred nanometers in diameter were present (Figure 2a). With the addition of DMSO, the structures collapsed and shrank until peptoid fibers with spheres attached to the fiber edges formed at 5 days (Figure 2b,c). Also present at 5 days were small, amorphous sheets. The fibers and sheets grew slightly over the course of a few weeks but never formed crystalline sheets, as evidenced by their irregularity in size, shape, and contrast in TEM (Figure 2d). Given that assembled structures were present immediately after dissolution, we believe the observed structural evolution is based on peptoid monomer rearrangement rather than complete dissolution and re-assembly from monomers, as seen in aqueous conditions. In general, these observations suggest hindered assembly in the organic solvent system, likely due to decreased solubility of the monomer units. 

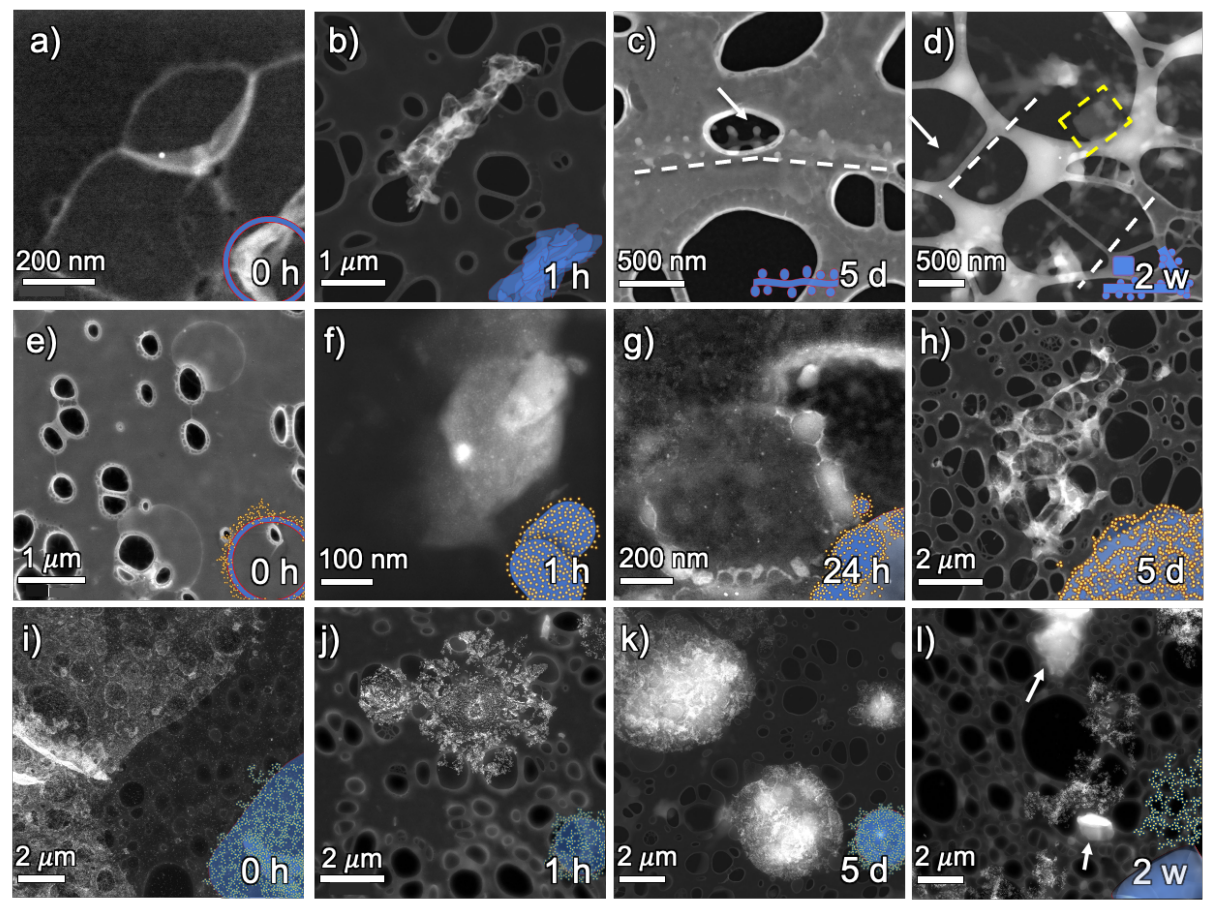

Figure 2. Peptoid assembly in $\mathrm{CHCl}_{3} / \mathrm{DMSO}$ alone (a-d), with MEEAA-CdSe (e-h), and with OA-CdSe (i-1). Peptoid alone showed large hollow spheres (a) that collapsed to form large aggregates (b), and eventually fibers (white dashed line, c) decorated by small dense spheres (arrow, c). Some sheet formation was observed (yellow dashed line) but no large, well-defined sheets were observed (d). The presence of MEEAA-CdSe QDs produced similar large hollow spheres decorated by QDs prior to DMSO addition (e), but then facilitated the solubilization of the peptoid, generating sheets with an even distribution of QDs(f). Over time QDs concentrated at the edges $(\mathrm{g})$, ultimately resulting in assembly of the small sheets into large multilayer hybrid sheets similar to what was observed in $\mathrm{MeCN} / \mathrm{H}_{2} \mathrm{O}$ (h). OA-CdSe generated a hydrophobic hybrid monomer unit that formed large 2D sheets in $\mathrm{CHCl}_{3}$ (i). Upon addition of DMSO collapse to form small 2D aggregates $(\mathrm{j})$ that evolved into 3D hybrid multilayers $(\mathrm{k})$, and eventually phase segregation of peptoid (white arrows) and QD (1).

Adding QDs with high coverage of an amphiphilic ligand should increase the solubility of the hybrid monomer unit and facilitate the formation of sheets. MEEAA-CdSe was assembled with 1 eq. of peptoid using the organic solvent system. Prior to the addition of DMSO, the QDs did not alter the hollow peptoid structures, but were instead bound to the exterior (Figure 2e). After the addition of DMSO, small peptoid sheets evenly decorated with QDs rapidly appeared and underwent aggregation over time (Figure 2f,g). The hybrid structures formed over 4 weeks primarily included large assemblies composed of smaller sheets with a higher density of QDs on the edges (Figure 2h), similar to the assemblies observed in $\mathrm{MeCN} / \mathrm{H}_{2} \mathrm{O}$. The assembly of hybrid monomer units in organic solvents did not produce spherical and fiber intermediates as in $\mathrm{MeCN} / \mathrm{H}_{2} \mathrm{O}$ (Figure 1f-h) but proceeded directly to hybrid peptoid-QD sheets after only $1 \mathrm{~h}$. The QDs were again seen to etch to $1.72 \pm 0.22 \mathrm{~nm}$, as was observed in the aqueous system. This more rapid progression to the hybrid sheet structures suggests that the QD-peptoid monomer unit is more soluble than the peptoid alone in the organic solvents, resulting in greater lability and faster assembly. Interestingly, these observations are opposite to the trends observed in the 
aqueous system where additional intermediates and slower sheet formation were noted in the case of the QDs. Together these data suggest that monomer solubility plays a crucial role in the assembly dynamics and that this solubility can be dramatically tuned based on the ligation of the appended QDs.

To further push the assembly pathway away from evaporative ordering based on hydrophobicity, OA-CdSe QDs were assembled with 1 eq. of peptoid. A hybrid unit with OACdSe yields a highly hydrophobic unit with both the QD surface and the exposed portion of the peptoid being insoluble in DMSO. Prior to DMSO addition, large 2D hybrid sheets were seen after $18 \mathrm{~h}$ in $\mathrm{CHCl}_{3}$ with lengths greater than $15 \mu \mathrm{m}$ (Figure 2i). Within the sheets there were areas of folding or curling, but they appeared to be primarily a monolayer of QDs without any distinct ordering among the QDs (Figure S11). This sheet formation indicates that by changing the QD surface chemistry, assembly can be induced without the addition of an antisolvent and without any evaporative processes as a driving force. With the addition of DMSO, the sheets collapsed initially into 2D circular structures that grew into larger 3D aggregates over time (Figure 2j,k). We hypothesize that this 3D aggregation is due to the frustrated structure where neither the QDs nor peptoids are sufficiently soluble in the $\mathrm{CHCl}_{3} / \mathrm{DMSO}$ solvent mixture, resulting in amorphous packing of the hydrophobic units. After most of the $\mathrm{CHCl}_{3}$ evaporated, phase segregation was observed between the peptoids and QDs with large peptoid-rich structures forming and free QDs scattered across the TEM grid (Figure 2l). This phase segregation suggests that the driving force for peptoids to assemble is still present under these conditions, but the peptoids are unable to order in a favorable manner with the QDs attached.

\section{QD-mediated synthesis of hybrid structures and binary-QD lattices.}

Another route to change the peptoid-QD assembly is by changing the size of the QD relative to the peptoid and increasing the overall bulk of the hybrid monomer unit. CdS QDs were synthesized with OA capping ligands and then were subjected to ligand exchange to produce $5.1 \mathrm{~nm}$ MEEAA-CdS QDs. These larger QDs have similar reactivity and surface chemistry to the MEEAA-capped CdSe QDs but have much more distinct and well-defined surface facets due to the increase in size. The same general $\mathrm{CHCl}_{3} / \mathrm{DMSO}$ assembly process was followed as above with one peptoid monomer per QD, with the only notable difference being the overall volume of QD was much larger than previously due to the increased QD size. In $\mathrm{CHCl}_{3}$, large, amorphous peptoid aggregates were seen with QDs on the edges (Figure S12a). QDs were seen to order into a linear packing pattern near the edges of the peptoid aggregates (Figure $\mathbf{S 1 2 b , c ) . ~ E x p o s u r e ~ t o ~ t h e ~ p e p t o i d s ~ l e d ~ t o ~ a n ~ i n i t i a l ~ e t c h i n g ~ o f ~ t h e ~ Q D s ~ t o ~ g i v e ~ a ~ d i a m e t e r ~ o f ~} 3.50$ $\pm 0.37 \mathrm{~nm}$. These peptoid aggregates with linear ordered QDs on the edges were maintained after DMSO addition with the appearance of large spherical aggregates comprised of primarily QDs, likely due to the high QD coverage (Figure 3a). By $24 \mathrm{~h}$, large 2D sheets were seen on the order of $10 \mu \mathrm{m}$ that were persistent through the rest of the evaporation process (Figure $3 \mathbf{b}$ ). Closer inspection of the sheets revealed the QDs packing in a square close packed array, with one QD having four nearest neighbors (Figure 3c). This ordering was observed in patches throughout the sheets with crystalline domain sizes ranging from 15 to $100 \mathrm{~nm}$ (Figure S13). These sheets were noticeably distinct from other assemblies we had observed previously and are reminiscent of structures that are dictated by inter-QD interactions arising from oriented attachment (vide infra), with the peptoid acting as a surface modifier ${ }^{49-52}$. This indicates that having a large pendant group relative to length of the peptoid disrupts the expected inter-peptoid interdigitation, preventing the formation of standard peptoid sheets. 

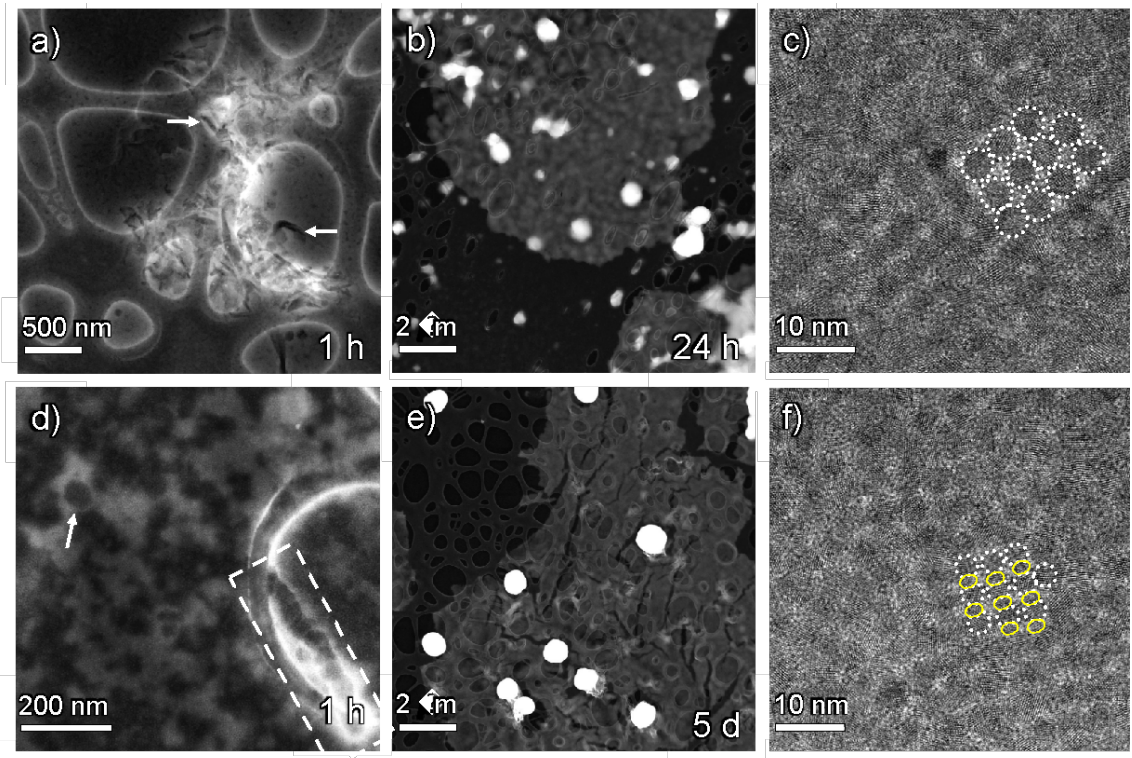

Figure 3. Assembly of CdS QDs with 1 eq. peptoid at $1 \mathrm{~h}$ (a) and $24 \mathrm{~h}$ (b). Early time points show phase segregation with rectangular peptoid structures (white arrow) decorated with QDs (a) until peptoid-QD integrated sheets form ( $24 \mathrm{~h}$ ). Hybrid sheet shows CdS QDs (white dotted line) in square close packed arrays (c). Assembly of CdS:CdSe (1:1) QDs with 1 eq. peptoid at $1 \mathrm{~h}$ (d) and $5 \mathrm{~d}(\mathrm{e})$. Amorphous peptoid structures (white dashed line) and micelles (white arrow) decorated with QDs are seen at $1 \mathrm{~h}$ until sheets form after $5 \mathrm{~d}$ (e). The same square close packed arrays are seen for the mixed system (f) with CdSe QDs (yellow circles) seen between the CdS QDs (white dotted lines).

Ordered nanoparticle superlattices with multiple types of nanoparticles have been explored through slow evaporation on a surface or at an interface, as well as by functionalization with ligands with chemical complementarity like DNA ${ }^{11,19,53,54}$. In the systems based on evaporation, the nanoparticle packing can be altered by changing the sizes of the nanoparticles relative to each other and their relative stoichiometries ${ }^{54}$. We sought to combine the CdSe and CdS QD systems to access free-standing binary QD assemblies. A total of 1 eq. of peptoid per total QD was combined with $\mathrm{CdSe}$ and $\mathrm{CdS}(1: 1)$ and assembled in $\mathrm{CHCl}_{3}$ and DMSO. The general assembly pathway was consistent with the CdS system, but sheet generation was slightly slowed. Peptoid micelles, previously seen in the CdSe system, were also observed at early time points with QDs in a linear pattern near the edges (Figure 3d). The QDs were dominated by $\mathrm{CdS}$, with CdSe being relatively difficult to identify, but were observed throughout the time series with the identification of $\sim 2 \mathrm{~nm}$ particles (Figure S14). We propose that this dominating effect of the CdS is a result of the more well-defined faceting and greater total volume of CdS present compared to CdSe. Over the course of 4 weeks, the sheets grew while remaining 2D until at 4 weeks ordering was observed within the sheets in a similar square close packed pattern as seen with CdS QDs alone (Figure 3e,f). When CdS and CdSe were mixed without peptoid present, there was no evidence of ordering between the QDs (Figure S15). Some aggregation of the QDs was seen due to high QD concentration but no free standing, 2D structures were observed in $\mathrm{CHCl}_{3}$ or $\mathrm{CHCl}_{3} / \mathrm{DMSO}$. Increasing the ratio of $\mathrm{CdSe}$ to $\mathrm{CdS}$ did not lead to different packing structures, but rather a slow transformation of large sheets to smaller peptoidQD sheets attached via bridging QDs. With increasing CdSe:CdS, the packing became more 
disordered, moving from square-packed QDs to linear packing, until total disorder was observed (Figure S16). These results suggest the CdSe acts as an "impurity" in the system, not dictating the structure as the $\mathrm{CdS}$ does. As the concentration of impurity increases, the sheets begin to break apart into smaller structures and aggregate in a fashion similar to that seen in the CdSe only system. Assembly of CdS and CdSe QDs into ordered arrays did not show significant changes in the optical properties. No change was observed in the location or intensity of the photoluminescence for the assemblies and a $2 \mathrm{~nm}$ redshift was seen for the absorbance (Figure S17). We hypothesize that the lack of long-range order and decreased solubility of the assemblies with time give properties comparable to free QDs.

\section{Characterization of $C d S$ and $C d S / C d S e$ ordered arrays and the role of peptoid.}

To understand the formation of these ordered $\mathrm{CdS}$ and $\mathrm{CdS} / \mathrm{CdSe}$ assemblies further, we sought to characterize their structure and dependence on drying through TEM image analysis, atomic force microscopy (AFM), and altering sample deposition. The porous spacing between the CdS particles was seen to be $1.75 \pm 0.20 \mathrm{~nm}$ via TEM (Figure 4a). For $3.5 \mathrm{~nm}$ particles (after etching), without accounting for ligands present, the pore spacing between particles would be expected to have a diameter of $1.4 \mathrm{~nm}$. Accounting for ligands on the QD surface that are $\sim 1.5$ $\mathrm{nm}$ in length with minimal interdigitation between neighboring ligand shells, a spacing of $1.9 \mathrm{~nm}$ would be expected (Figure 4b). The packing for mixed QDs showed a slight decrease in the pore size via TEM (1.64 $\pm 0.20 \mathrm{~nm}$ ) compared to CdS QD assemblies (Figure 4e). Interestingly, an apparent necking feature was seen between the CdS QDs in the mixed QD samples with a width of $1.65 \pm 0.25 \mathrm{~nm}$, consistent with the diameter of the etched CdSe QDs (Figure 3f, yellow dashed lines). This feature did not appear to be consistent with particle fusion due to discontinuity of the lattice fringes and its round instead of tapered appearance, but rather the presence of a separate CdSe sublattice. Identification of the CdSe QDs was confirmed using high-resolution TEM to show d spacing values of $0.35 \mathrm{~nm}$ and $0.23 \mathrm{~nm}$, consistent with (111) and (220) planes of zinc-blende CdSe (Figure 4e). If the CdSe were positioned in the plane with the CdS, accounting for partial interdigitation of the ligand shells, we would expect an increase in pore size to $2.3 \mathrm{~nm}$ (Figure 4c). However, positioning the CdSe above or below the plane of $\mathrm{CdS}$ at interstitial sites results in the lattice of $\mathrm{CdS}$ being preserved with an apparent reduction in the pore size $(1.6 \mathrm{~nm})$ due to additional material at the interstitial sites (Figure 4d). 


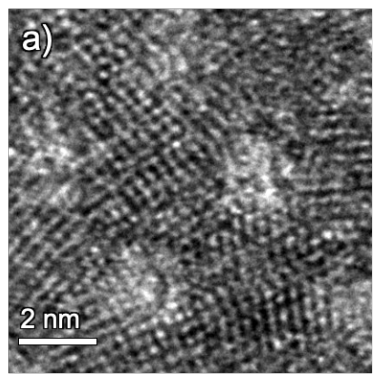

b)
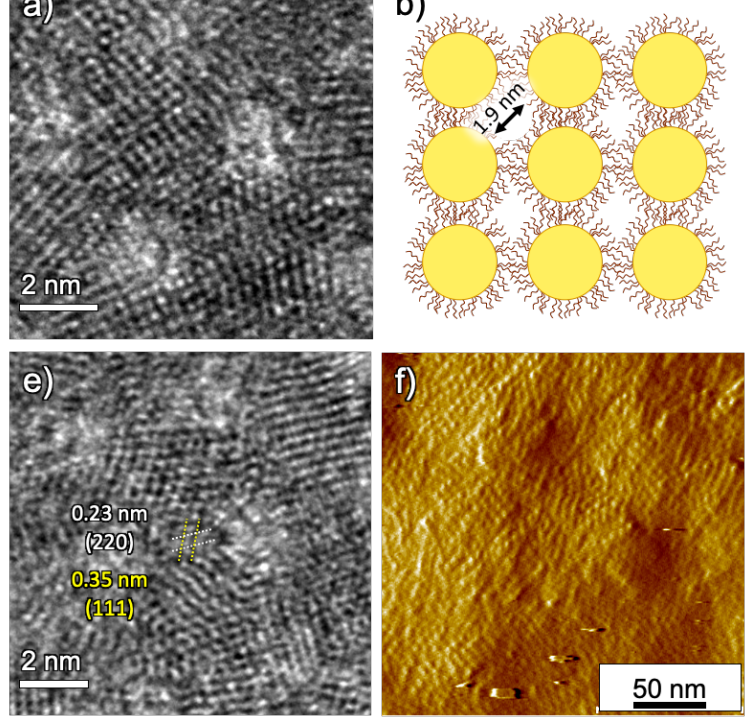
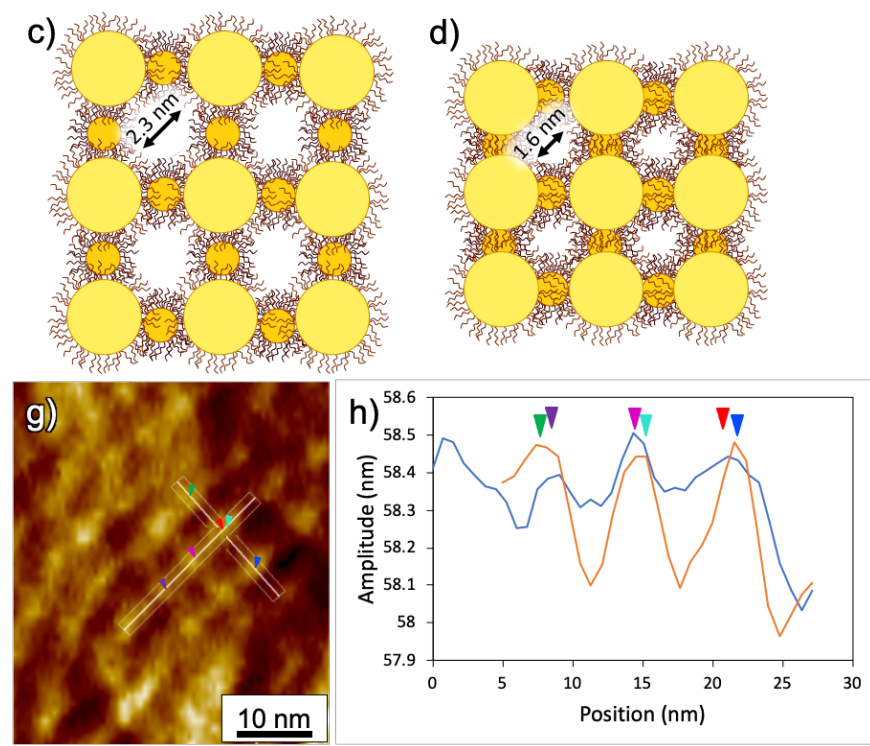

Figure 4. High-resolution image of CdS QDs within the 2D sheets showing a pore diameter of $1.75 \pm 0.20 \mathrm{~nm}$ (a). Carton representation of CdS QDs with minimal interdigitation of the ligands gives an expected pore diameter of $1.9 \mathrm{~nm}$ (b). Cartoon representation of CdS QDs with incorporated CdSe QDs between without overlap (c) and with overlap (d) giving an expected pore diameter of $2.3 \mathrm{~nm}$ and $1.6 \mathrm{~nm}$, respectively. High-resolution image within the sheet of mixed $\mathrm{CdS}$ and $\mathrm{CdSe}$ shows a feature between the CdS with a width of $1.65 \pm 0.25 \mathrm{~nm}$ and pore size of $1.64 \pm 0.20 \mathrm{~nm}(\mathrm{e})$. The feature showed lattice spacing of $0.35 \mathrm{~nm}$ and $0.23 \mathrm{~nm}$, consistent with the (111) and (220) planes of CdSe. AFM amplitude image of the mixed sheets showing a patchy structure with ordered QDs (f). Perpendicular line scans of the mixed lattice show one direction with only CdS (orange line) and one with intermediate peaks (blue), presumably from out of plane $\mathrm{CdSe}(\mathrm{g}, \mathrm{h})$.

Additional characterization of the assemblies was done via AFM to confirm the QD ordering and determine the position of CdSe relative to CdS. Due to the large size of the sheets, the edge of the sheet was not identified with AFM. Nevertheless, the surface of the sheet showed a patchy structure with ordering throughout (Figure 4f). The patches on the surface showed a layered structure with many layers exhibiting square close packed ordering (Figure S18). We hypothesize that these assemblies are highly ordered in two dimensions but lack registry in the $\mathrm{Z}$ direction due to the overlap of the sheets. AFM images showed the CdSe are not in plane with the CdS as no small QDs were observed (Figure 4g-h, orange line). However, line scans of the ordered assemblies showed an intermediate peak between CdS, presumably from a layer of CdSe QDs offset from the top layer (Figure 4g-h, blue line).

Square close packing has been observed extensively for lead chalcogenide QDs both through fusion and drying effects due to decreased ligation on specific facets ${ }^{49-52}$. It has been shown that this fusion process occurs through oriented attachment under conditions in which ligand coverage on the (100) facet is reduced ${ }^{49-52,55}$. This oriented packing is not observed for QDs smaller than $4 \mathrm{~nm}$ as the (100) facet is not present in the smaller nanoparticles ${ }^{52,55}$. When the particles are not fused, these lattices arise due to drying effects. In our system, if the peptoids were acting as isolated ligands on the surface of the CdS QDs and only the QDs dictated the structure, one would expect the sheets to arise due to similar drying effects, not the observed solution-phase assembly. To confirm the sheets form in solution due to peptoid and QD 
contributions, the sheets were isolated by slow centrifugation of the solution, producing a yellow precipitate that was resuspended in DMSO. Large sheets formed in solution would be expected to settle out under these conditions with any free QDs remaining in solution. TEM analysis showed the same 2D sheets in the pellet with slight destabilization seen by a reduction in the percentage of ordered arrays (Figure S19). The supernatant only showed structures associated with random aggregation of free QDs and no evidence of ordering within the aggregates. These results indicate that large structures are present in solution and resemble the large sheets present in aliquots taken from solution. To further determine if the sheets are present in solution or result from a drying effect, the TEM grid sample preparation method was altered. To disrupt any drying effects due to slow evaporation, the sample was drop cast onto the grid and let settle for 2 min, dabbed off, and immediately placed under vacuum to rapidly dry the sample. In contrast, typical samples are allowed to settle for $15 \mathrm{~min}$ before dabbing off the grid and allowing to dry on a Kimwipe for an additional $15 \mathrm{~min}$ before exposure to the vacuum. Analysis of the rapidly dried grid still showed the same large 2D sheets with square close packed QDs, as seen previously (Figure S20). Moreover, the prevalence of large QD aggregates was decreased and the sheets appeared thinner at the edges relative to standard sample preparation, implying that faster drying of the sample reduces overcrowding and stacking of free QDs on the grid.

Finally, to determine if the assemblies form because of bottom-up assembly and integration of QDs into the sheets or decoration of QDs onto a pre-formed peptoid surface, 1:1 $\mathrm{CdS}$ and CdSe QDs were added to pre-formed peptoid sheets in DMSO and allowed to incubate at room temperature. Upon initial addition, QDs were seen to stick non-uniformly to peptoid surfaces, with the sheets mostly remaining intact (Figure S21a). Over the course of 2 weeks, large sheets similar to bottom-up assembly were observed but did not show ordering of the QDs and stability was lost over time to produce small, rounded peptoid sheets with uneven QD distribution (Figure S21b-e). These results indicate that the hybrid sheets are not formed due to drying effects alone and the QD superlattices do not arise from post-synthetic coordination to assembled peptoid sheets. Instead, these results support a hybrid system wherein the QDs and peptoids assemble in solution together as a hybrid unit from an integrated structure. We hypothesize that the peptoids are bound to the QD surface, acting as a ligand rather than an assembled template. On the QD surface the peptoid monomers can act as a linker between dots or bind specific facets, leading to the observed square packed superlattices where the inter-QD interactions dictate the final structure.

\section{Conclusions.}

To access new hierarchical hybrid materials, we must first understand the design principles that dictate the evolution of structure in these dynamic systems and determine how different parameters can be used to tune the reaction landscape. In this work, we have outlined two assembly regimes between QDs and peptoids that reversibly bind to the QD surface. In the first regime, the hybrid structures are controlled by both the peptoid and QD, whereas in the second regime, the hybrid structures are dominated by inter-QD interactions. The assembly pathway and progression of intermediates for peptoid-QD hybrid structures were studied in aqueous and organic conditions. Attachment of the QD to the peptoid altered the solubility of the hybrid monomer unit aiding the assembly in organic conditions and stabilizing intermediates in the aqueous system. Design rules such as the ratio of peptoid monomer relative to QD, hydrophobicity of the QD, and size of the QD were explored. Changing the equivalents of the peptoid at low concentrations only changed the speed of assembly based on the diffusion 
properties of the peptoid-QD monomer unit. Altering the QD capping ligand changed the solubility of the hybrid peptoid-QD monomer unit, generating 2D assemblies prior to antisolvent addition and disrupting sheet formation afterwards. With larger faceted CdS QDs, it was found that the QD dictated the structure, resulting in highly ordered square packed arrays with peptoids acting as a surface ligand on the QD. Combining two different sized QDs allowed for the incorporation of the smaller dots on top of the CdS lattice, with structures still being dictated by the large CdS QDs. Understanding these design elements allows for careful planning and manipulation of QD size, surface chemistry, peptoid sequence, and solvents to tune access to specific hybrid architectures and predictably generate structural complexity.

\section{Supporting Information.}

Electronic supplementary information (ESI) available: Complete experimental methods and additional figures including TEM images, EDX, UV-Vis, and NMR spectroscopy data. See DOI: XXXXXX.

\section{Corresponding Author. \\ *cossairt@uw.edu}

\section{Funding Sources.}

US Department of Energy, Office of Science, Office of Basic Energy Sciences DE-SC0019288.

\section{Acknowledgements.}

This material is based upon work supported by the U.S. Department of Energy, Office of Science, Office of Basic Energy Sciences as part of the Energy Frontier Research Centers program: CSSAS-The Center for the Science of Synthesis Across Scales under award number DE-SC0019288. Peptoid design and synthesis were performed at the Department of Energy's Pacific Northwest National Laboratory (PNNL). PNNL is a multi-program national laboratory operated for Department of Energy by Battelle under Contract No. DE-AC05-76RL01830. Part of this work was conducted at the Molecular Analysis Facility, a National Nanotechnology Coordinated Infrastructure site at the University of Washington which is supported in part by the National Science Foundation (grant NNCI-1542101), the University of Washington, the Molecular Engineering \& Sciences Institute, and the Clean Energy Institute.

\section{References.}

1. Pigliacelli, C.; Sánchez-Fernández, R.; García, M. D.; Peinador, C.; Pazos, E. SelfAssembled Peptide-Inorganic Nanoparticle Superstructures: From Component Design to Applications. Chem. Commun. 2020, 56 (58), 8000-8014.

2. Levin, A.; Hakala, T. A.; Schnaider, L.; Bernardes, G. J. L.; Gazit, E.; Knowles, T. P. J. Biomimetic Peptide Self-Assembly for Functional Materials. Nature Rev. Chem. 2020, 4 (11), 615-634.

3. Saveleva, M. S.; Eftekhari, K.; Abalymov, A.; Douglas, T. E. L.; Volodkin, D.; Parakhonskiy, B. V.; Skirtach, A. G. Hierarchy of Hybrid Materials-The Place of Inorganics-in-Organics in It, Their Composition and Applications. Front. Chem. 2019, 7, 179.

4. Li, Z.; Fan, Q.; Yin, Y. Colloidal Self-Assembly Approaches to Smart Nanostructured Materials. Chem. Rev. 2021, DOI: 10.1021/acs.chemrev.1c00482. 
5. Kuzuya, A.; Ohya, Y. DNA Nanostructures as Scaffolds for Metal Nanoparticles. Polym. J. 2012, 44 (6), 452-460.

6. Yamauchi, M.; Masuo, S. Self-Assembly of Semiconductor Quantum Dots Using Organic Templates. Chem. Eur. J. 2020, 26 (32), 7176-7184.

7. Chen, C.-L.; Rosi, N. L. Peptide-Based Methods for the Preparation of Nanostructured Inorganic Materials. Angew. Chem. Int. Ed. 2010, 49 (11), 1924-1942.

8. Ritchhart, A.; Monahan, M.; Mars, J.; Toney, M. F.; De Yoreo, J. J.; Cossairt, B. M. Covalently Linked, Two-Dimensional Quantum Dot Assemblies. Langmuir 2020, 36 (33), 9944-9951.

9. Cai, B.; Li, Z.; Chen, C.-L. Programming Amphiphilic Peptoid Oligomers for Hierarchical Assembly and Inorganic Crystallization. Acc. Chem. Res. 2021, 54 (1), 8191.

10. Hoheisel, T. N.; Hur, K.; Wiesner, U. B. Block Copolymer-Nanoparticle Hybrid SelfAssembly. Prog. Polym. Sci. 2015, 40, 3-32.

11. Macfarlane, R. J.; Lee, B.; Jones, M. R.; Harris, N.; Schatz, G. C.; Mirkin, C. A. Nanoparticle Superlattice Engineering with DNA. Science 2011, 334, 204-208.

12. Tian, Y.; Lhermitte, J. R.; Bai, L.; Vo, T.; Xin, H. L.; Li, H.; Li, R.; Fukuto, M.; Yager, K. G.; Kahn, J. S.; Xiong, Y.; Minevich, B.; Kumar, S. K.; Gang, O. Ordered ThreeDimensional Nanomaterials Using DNA-Prescribed and Valence-Controlled Material Voxels. Nature Mater. 2020, 19 (7), 789-796.

13. Zheng, J.; Constantinou, P. E.; Micheel, C.; Alivisatos, A. P.; Kiehl, R. A.; Seeman, N. C. Two-Dimensional Nanoparticle Arrays Show the Organizational Power of Robust DNA Motifs. Nano Lett. 2006, 6 (7), 1502-1504.

14. Fry, H. C.; Silveira, G. de Q.; Cohn, H. M.; Lee, B. Diverse Bilayer Morphologies Achieved via $\alpha$-Helix-to- $\beta$-Sheet Transitions in a Short Amphiphilic Peptide. Langmuir 2019, 35 (27), 8961-8967.

15. Lin, Y.; Penna, M.; Thomas, M. R.; Wojciechowski, J. P.; Leonardo, V.; Wang, Y.; Pashuck, E. T.; Yarovsky, I.; Stevens, M. M. Residue-Specific Solvation-Directed Thermodynamic and Kinetic Control over Peptide Self-Assembly with 1D/2D Structure Selection. ACS Nano 2019, 13 (2), 1900-1909.

16. Ozkan, A. D.; Tekinay, A. B.; Guler, M. O.; Tekin, E. D. Effects of Temperature, PH and Counterions on the Stability of Peptide Amphiphile Nanofiber Structures. RSC Adv. 2016, 6 (106), 104201-104214.

17. Zhang, S.; Alberstein, R. G.; De Yoreo, J. J.; Tezcan, F. A. Assembly of a Patchy Protein into Variable 2D Lattices via Tunable Multiscale Interactions. Nature Commun. 2020, 11 (1), 3770 .

18. Jiao, F.; Wu, X.; Jian, T.; Zhang, S.; Jin, H.; He, P.; Chen, C.-L.; De Yoreo, J. J. Hierarchical Assembly of Peptoid-Based Cylindrical Micelles Exhibiting Efficient Resonance Energy Transfer in Aqueous Solution. Angew. Chem. Int. Ed. 2019, 58 (35), 12223-12230.

19. Shevchenko, E. V.; Talapin, D. V.; Kotov, N. A.; O’Brien, S.; Murray, C. B. Structural Diversity in Binary Nanoparticle Superlattices. Nature 2006, 439 (7072), 55-59.

20. Lunn, D. J.; Finnegan, J. R.; Manners, I. Self-Assembly of "Patchy" Nanoparticles: A Versatile Approach to Functional Hierarchical Materials. Chem. Sci. 2015, 6 (7), 36633673. 
21. Boles, M. A.; Talapin, D. V. Binary Assembly of PbS and Au Nanocrystals: Patchy PbS Surface Ligand Coverage Stabilizes the CuAu Superlattice. ACS Nano 2019, 13 (5), 5375-5384.

22. Lee, H.-Y.; Shin, S. H. R.; Drews, A. M.; Chirsan, A. M.; Lewis, S. A.; Bishop, K. J. M. Self-Assembly of Nanoparticle Amphiphiles with Adaptive Surface Chemistry. ACS Nano 2014, 8 (10), 9979-9987.

23. Abécassis, B.; Tessier, M. D.; Davidson, P.; Dubertret, B. Self-Assembly of CdSe Nanoplatelets into Giant Micrometer-Scale Needles Emitting Polarized Light. Nano Lett. 2014, 14 (2), 710-715.

24. Santos, P. J.; Cheung, T. C.; Macfarlane, R. J. Assembling Ordered Crystals with Disperse Building Blocks. Nano Lett. 2019, 19 (8), 5774-5780.

25. Santos, P. J.; Cao, Z.; Zhang, J.; Alexander-Katz, A.; Macfarlane, R. J. Dictating Nanoparticle Assembly via Systems-Level Control of Molecular Multivalency. J. Am. Chem. Soc. 2019, 141 (37), 14624-14632.

26. Ni, B.; Fu, C.; Pan, S.; He, L.; Lin, Z.; Peng, J. Semiconducting Spaghetti-like OrganicInorganic Nanojunctions via Sequential Self-Assembly of Conjugated Polymers and Quantum Dots. Chem. Mater. 2022, 34 (2), 847-853.

27. Kubo, N.; Yamauchi, M.; Yamamoto, S.; Masuo, S. Elucidation of the Mechanism of Quantum Dot Arrangement Based on Self-Assembly of an Azobenzene Derivative. Bull. Chem. Soc. Jpn. 2021, 94 (6), 1799-1803.

28. DeStefano, A. J.; Segalman, R. A.; Davidson, E. C. Where Biology and Traditional Polymers Meet: The Potential of Associating Sequence-Defined Polymers for Materials Science. JACS Au 2021, 1 (10), 1556-1571.

29. Li, Z.; Cai, B.; Yang, W.; Chen, C.-L. Hierarchical Nanomaterials Assembled from Peptoids and Other Sequence-Defined Synthetic Polymers. Chem. Rev. 2021, 121 (22), 14031-14087.

30. Sun, J.; Zuckermann, R. N. Peptoid Polymers: A Highly Designable Bioinspired Material. ACS Nano 2013, 7 (6), 4715-4732.

31. Jin, H.; Ding, Y.-H.; Wang, M.; Song, Y.; Liao, Z.; Newcomb, C. J.; Wu, X.; Tang, X.Q.; Li, Z.; Lin, Y.; Yan, F.; Jian, T.; Mu, P.; Chen, C.-L. Designable and Dynamic Single-Walled Stiff Nanotubes Assembled from Sequence-Defined Peptoids. Nature Commun. 2018, 9 (1), 270.

32. Jin, H.; Jiao, F.; Daily, M. D.; Chen, Y.; Yan, F.; Ding, Y.-H.; Zhang, X.; Robertson, E. J.; Baer, M. D.; Chen, C.-L. Highly Stable and Self-Repairing Membrane-Mimetic 2D Nanomaterials Assembled from Lipid-like Peptoids. Nature Commun. 2016, 7 (1), 12252.

33. Wu, C. W.; Sanborn, T. J.; Huang, K.; Zuckermann, R. N.; Barron, A. E. Peptoid Oligomers with $\alpha$-Chiral, Aromatic Side Chains: Sequence Requirements for the Formation of Stable Peptoid Helices. J. Am. Chem. Soc. 2001, 123 (28), 6778-6784.

34. Robertson, E. J.; Battigelli, A.; Proulx, C.; Mannige, R. V.; Haxton, T. K.; Yun, L.; Whitelam, S.; Zuckermann, R. N. Design, Synthesis, Assembly, and Engineering of Peptoid Nanosheets. Acc. Chem. Res. 2016, 49 (3), 379-389.

35. Sun, J.; Jiang, X.; Lund, R.; Downing, K. H.; Balsara, N. P.; Zuckermann, R. N. SelfAssembly of Crystalline Nanotubes from Monodisperse Amphiphilic Diblock Copolypeptoid Tiles. Proc. Natl. Acad. Sci. 2016, 113 (15), 3954-3959. 
36. Xuan, S.; Jiang, X.; Spencer, R. K.; Li, N. K.; Prendergast, D.; Balsara, N. P.; Zuckermann, R. N. Atomic-Level Engineering and Imaging of Polypeptoid Crystal Lattices. Proc. Natl. Acad. Sci. 2019, 116 (45), 22491-22499.

37. Hammons, J. A.; Baer, M. D.; Jian, T.; Lee, J. R. I.; Weiss, T. M.; De Yoreo, J. J.; Noy, A.; Chen, C.-L.; Van Buuren, A. Early-Stage Aggregation and Crystalline Interactions of Peptoid Nanomembranes. J. Phys. Chem. Lett. 2021, 12 (26), 6126-6133.

38. Ma, X.; Zhang, S.; Jiao, F.; Newcomb, C. J.; Zhang, Y.; Prakash, A.; Liao, Z.; Baer, M. D.; Mundy, C. J.; Pfaendtner, J.; Noy, A.; Chen, C.-L.; De Yoreo, J. J. Tuning Crystallization Pathways through Sequence Engineering of Biomimetic Polymers. Nature Mater. 2017, 16 (7), 767-774.

39. Wang, M.; Song, Y.; Zhang, S.; Zhang, X.; Cai, X.; Lin, Y.; Yoreo, J. J. D.; Chen, C.-L. Programmable Two-Dimensional Nanocrystals Assembled from POSS-Containing Peptoids as Efficient Artificial Light-Harvesting Systems. Sci. Adv. 2021, 7 (20), No. eabg1448.

40. Yan, F.; Liu, L.; Walsh, T. R.; Gong, Y.; El-Khoury, P. Z.; Zhang, Y.; Zhu, Z.; De Yoreo, J. J.; Engelhard, M. H.; Zhang, X.; Chen, C.-L. Controlled Synthesis of HighlyBranched Plasmonic Gold Nanoparticles through Peptoid Engineering. Nature Commun. 2018, 9 (1), 2327.

41. Monahan, M.; Cai, B.; Jian, T.; Zhang, S.; Zhu, G.; Chen, C.-L.; Yoreo, J. J. D.; Cossairt, B. M. Peptoid-Directed Assembly of CdSe Nanoparticles. Nanoscale 2021, 13 (2), 1273 1282.

42. Ma, J.; Cai, B.; Zhang, S.; Jian, T.; De Yoreo, J. J.; Chen, C.-L.; Baneyx, F. Nanoparticle-Mediated Assembly of Peptoid Nanosheets Functionalized with SolidBinding Proteins: Designing Heterostructures for Hierarchy. Nano Lett. 2021, 21 (4), 1636-1642.

43. Merrill, N. A.; Yan, F.; Jin, H.; Mu, P.; Chen, C.-L.; Knecht, M. R. Tunable Assembly of Biomimetic Peptoids as Templates to Control Nanostructure Catalytic Activity. Nanoscale 2018, 10 (26), 12445-12452.

44. Yang, W.; Yin, Q.; Chen, C.-L. Designing Sequence-Defined Peptoids for Biomimetic Control over Inorganic Crystallization. Chem. Mater. 2021, 33 (9), 3047-3065.

45. Knauf, R. R.; Lennox, J. C.; Dempsey, J. L. Quantifying Ligand Exchange Reactions at CdSe Nanocrystal Surfaces. Chem. Mater. 2016, 28 (13), 4762-4770.

46. Bera, D.; Qian, L.; Tseng, T.-K.; Holloway, P. H. Quantum Dots and Their Multimodal Applications: A Review. Materials 2010, 3 (4), 2260-2345.

47. Huang, Y.; Cohen, T. A.; Sperry, B. M.; Larson, H.; Nguyen, H. A.; Homer, M. K.; Dou, F. Y.; Jacoby, L. M.; Cossairt, B. M.; Gamelin, D. R.; Luscombe, C. K. Organic Building Blocks at Inorganic Nanomaterial Interfaces. Mater. Horiz. 2022, 9 (1), 61-87.

48. Mussa Farkhani, S.; Valizadeh, A. Review: Three Synthesis Methods of CdX (X = Se, S or Te) Quantum Dots. IET Nanobiotechnology 2014, 8 (2), 59-76.

49. Liu, J.; Enomoto, K.; Takeda, K.; Inoue, D.; Pu, Y.-J. Simple Cubic Self-Assembly of $\mathrm{PbS}$ Quantum Dots by Finely Controlled Ligand Removal through Gel Permeation Chromatography. Chem. Sci. 2021, 12 (30), 10354-10361.

50. Walravens, W.; De Roo, J.; Drijvers, E.; ten Brinck, S.; Solano, E.; Dendooven, J.; Detavernier, C.; Infante, I.; Hens, Z. Chemically Triggered Formation of TwoDimensional Epitaxial Quantum Dot Superlattices. ACS Nano 2016, 10 (7), 6861-6870. 
51. Balazs, D. M.; Dirin, D. N.; Fang, H.-H.; Protesescu, L.; ten Brink, G. H.; Kooi, B. J.; Kovalenko, M. V.; Loi, M. A. Counterion-Mediated Ligand Exchange for PbS Colloidal Quantum Dot Superlattices. ACS Nano 2015, 9 (12), 11951-11959.

52. Baumgardner, W. J.; Whitham, K.; Hanrath, T. Confined-but-Connected Quantum Solids via Controlled Ligand Displacement. Nano Lett. 2013, 13 (7), 3225-3231.

53. Si, K. J.; Chen, Y.; Shi, Q.; Cheng, W. Nanoparticle Superlattices: The Roles of Soft Ligands. Adv. Sci. 2018, 5 (1), 1700179.

54. Chen, Z.; O'Brien, S. Structure Direction of II-VI Semiconductor Quantum Dot Binary Nanoparticle Superlattices by Tuning Radius Ratio. ACS Nano 2008, 2 (6), 1219-1229.

55. Kessler, M. L.; Dempsey, J. L. Mapping the Topology of PbS Nanocrystals through Displacement Isotherms of Surface-Bound Metal Oleate Complexes. Chem. Mater. 2020, 32 (6), 2561-2571. 